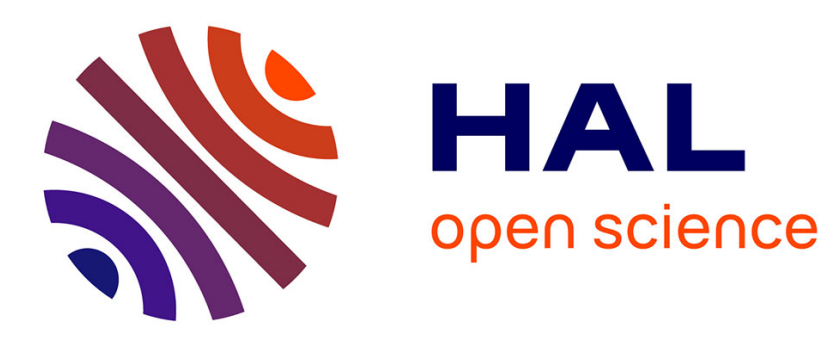

\title{
Periodic radio resource allocation to meet latency and reliability requirements in $5 \mathrm{G}$ networks
}

Yishu Han, Salah Eddine Elayoubi, Ana Galindo-Serrano, Vineeth Varma, Malek Messai

\section{- To cite this version:}

Yishu Han, Salah Eddine Elayoubi, Ana Galindo-Serrano, Vineeth Varma, Malek Messai. Periodic radio resource allocation to meet latency and reliability requirements in $5 \mathrm{G}$ networks. 87th Vehicular Technology Conference, VTC2018-Spring, Jun 2018, Porto, Portugal. hal-01812631

\section{HAL Id: hal-01812631 https://hal.science/hal-01812631}

Submitted on 11 Jun 2018

HAL is a multi-disciplinary open access archive for the deposit and dissemination of scientific research documents, whether they are published or not. The documents may come from teaching and research institutions in France or abroad, or from public or private research centers.
L'archive ouverte pluridisciplinaire HAL, est destinée au dépôt et à la diffusion de documents scientifiques de niveau recherche, publiés ou non, émanant des établissements d'enseignement et de recherche français ou étrangers, des laboratoires publics ou privés. 


\title{
Periodic Radio Resource Allocation to Meet Latency and Reliability Requirements in 5G Networks
}

\author{
Yishu Han*, Salah Eddine Elayoubi*, Ana Galindo-Serrano*, Vineeth S. Varma ${ }^{\dagger}$, Malek Messai* \\ * Orange Labs, Chatillon, France \\ $\dagger$ Universite de Lorraine, CRAN, UMR 7039, France
}

\begin{abstract}
Ultra-Reliable and Low Latency Communications (URLLC) is a challenging class of services to be supported by the fifth generation of mobile networks (5G). Among the URLLC services, many use cases, especially those related to factory automation, involve communications with relatively static radio conditions and a periodic generation of control or data packets. The transmission of these packets requires extremely low latency and ultra-reliable communication to enable realtime control of automation processes. In this paper, we discuss a mechanism of deterministic resource allocation to meet the URLLC requirement in terms of reliability and latency, including initial transmissions and controlled retransmissions. A joint resource allocation and modulation and coding schemes selection is performed so that the resource consumption is minimized, subject to latency and reliability constraints. We show that when applying the proposed resource allocation technique it is possible to achieve very low error rates.
\end{abstract}

\section{INTRODUCTION}

The 5th generation of mobile networks (5G) will not only support an evolution of traditional mobile communication services such as personal mobile broadband services; $5 \mathrm{G}$ will in addition support new use cases and enable a everything connected society [1]. These use cases are particularly for new Machine-Type Communications (MTC) services, including massive MTC and mission-critical MTC. The latter category requires communication with very high reliability, as well as very low latencies. One area in $5 \mathrm{G}$ that is considered increasingly important is, therefore, the capability to provide Ultra-Reliable and Low Latency Communications (URLLC), enabling new mission-critical MTC use cases [2]. Many verticals present interesting URLLC use cases and such as industry automation, tactile internet, virtual and augmented reality, smart grids, autonomous vehicles and drones. This large range of use cases presents different requirements in terms of latency and reliability. Namely, depending on the application the end to end latency can vary from 0.5 to 10 $\mathrm{ms}$ and the reliability from $10^{-3}$ to $10^{-5}$ or even fewer [10].

The URLLC framework enables real-time control and automation of dynamic processes in various fields, such as industrial process automation and manufacturing, which is the focus of this paper. Connecting machines in an industrial site enable many new opportunities for discrete manufacturing and help industrials to achieve a more efficient production. Today factory automation is largely based on wired connectivity. Among other standards, IEEE TSN (Time-Sensitive Networking) is increasingly used for ensuring deterministic communications protocol in industrial control applications. It is a set of Ethernet sub-standards that describe several mechanisms for guaranteed real-time delivery of Ethernet traffic. The core concept of TSN is deterministically scheduling traffic in queues through switched networks.

In order to provide more flexibility for the factories of the future, replacing wired communication links with wireless ones is foreseen in short to mid-term. Wireless communications in industrial venues are particularly due to their potential isolation and typical indoor environments. That is why the use of unlicensed spectrum has been proposed by numerous equipment vendors. This would allow the use of a proprietary infrastructure dedicated only to the industrial site wireless communications. Example of such systems is WirelessHART [3]. Nevertheless, the use of unlicensed spectrum will not guarantee communications without interference or spectrum crowding, which is of paramount importance when it comes to URLLC type of communications. We propose in this paper the usage of licensed 5G spectrum for URLLC, ensuring interference protection and full control of the operator.

We propose in this paper a joint Modulation and Coding Scheme (MCS) selection and resource allocation scheme for URLLC in factory automation use cases. Indeed, due to constraints in delay and reliability, sufficient radio resource should be provided, not only for the first transmission but also for retransmissions (within the delay budget). The choice of the MCS of the different users will impact the number of resources reserved for the first transmissions, but also the number of retransmissions. Indeed, a robust MCS will have a lower spectral efficiency, leading to a larger number of Resource Blocks (RBs) for sending the same applicative packet. However, this robust MCS will result in a lower packet loss probability and will necessitate a smaller amount of reserved RBs for retransmissions. We formulate an optimization problem where the MCS for each user is chosen so that the overall radio resource consumption is minimized, subject to latency and reliability constraints. The model takes as output detailed link level simulations that provide, for each modulation and channel coding scheme, the packet loss probability.

The remainder of this paper is structured as follows. In Section II we first introduce the system model along with our design considerations for an ultra-reliable and low-latency 


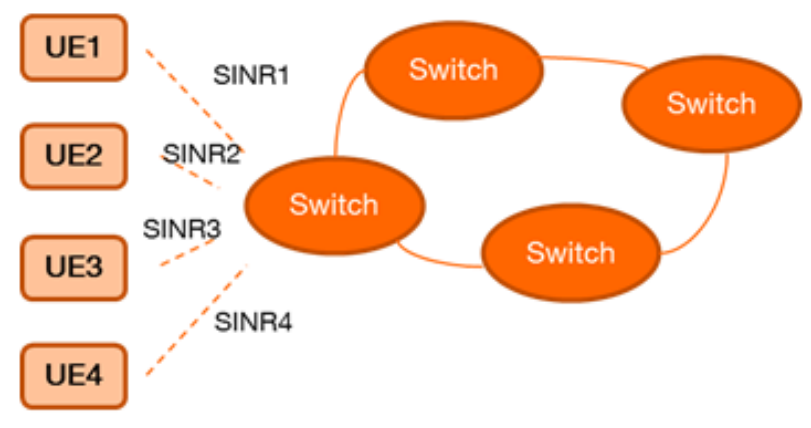

Fig. 1. System model

scheduling mechanism. Also, main simulation assumptions that are going to be used in the performance evaluations are presented. Section III provides the formalization of the network model to calculate radio resource consumption. In Section IV, the performance evaluations are presented with the detailed trade-off analysis in order to find the optimal solution. In Section V we discuss an extension of performance evaluation to meet per User Equipment (UE) requirement. Finally, Section VI concludes the article.

\section{SYSTEM MODEL}

Wired technologies, such as Ethernet-based systems, have been used for a long time for factory automation and they are still the dominant technologies in this field because of their reliability and real-time guarantee. However, the interest of using wireless communication for factory automation has grown recently thanks to the advantages in terms of flexible deployment and easy maintenance. The evolution in factory automation from wired to wireless communications should take place gradually and it is commonly agreed that the wires cannot be removed everywhere. In our study, we consider a mixed wired/wireless system within the factory, as illustrated in Fig. 1. In this figure, the main factory switches are fixed and connected via wires, while the last hop, i.e., the link between the UEs, integrated to the machines, and the switch is wireless.

The scheduling problem could also be divided into two parts: wired and wireless. We suppose that the TSN protocol, or equivalent, is applied in the wired network so that low and bounded jitter and deterministic end-to-end latency are ensured. Our study focuses on the wireless communication scheduling.

On the wireless side, UEs are characterized by different radio conditions so that their resource requirements are different and depend on their long-term Signal to Noise Ratios (SNRs) and on their chosen MCSs. Even if we suppose that the long-term SNRs are known, as machines are either fixed or characterized by a low mobility, their instantaneous radio conditions change due to fast fading, leading to some losses of packets. Retransmissions are then needed for recovering these lost packets, when possible. In our work, we adopt the Hybrid Automatic Repeat reQuest (HARQ), with Chase combining between packets. The whole system has to be designed so that the final packet loss rate is very low i.e., $10^{-5}$ ), with a minimal number of retransmissions in order to respect the low latency requirements (i.e., few milliseconds). A joint optimal resource allocation for first transmissions and retransmissions and MCS selections are then to be designed, as detailed in the following section.

\section{RADIO RESOURCE ALLOCATION SCHEME}

Our work targets (quasi) deterministic radio resource allocation mechanism over a wireless link. Radio resources are allocated into the time/frequency domain. In particular, in the time domain, they are allocated every Transmission Time Interval (TTI). In 4G, a TTI lasts for $1 \mathrm{~ms}$, while different TTI sizes are being defined for 5G. In the frequency domain, instead, the total bandwidth is divided in sub-channels ${ }^{1}$. A combination of a TTI and a subchannel is called RB and corresponds to the smallest radio resource unit that can be assigned to an UE for data transmission. In addition, a portion of the spectrum and certain time interval should be given to payloads of signaling. We will see, in the following section, how resource allocation can be done to achieve the URLLC requirements.

To guarantee deterministic scheduling, we propose that a periodic resource reservation is performed. Based on the average SNR, different MCSs are used for each user. The number of RBs that will be reserved can, therefore, be calculated. However, some packets will be lost with a userspecific packet error rate that depends on the chosen MCS and the SNR. An additional amount of RBs should thus be reserved for retransmissions and, if a retransmission occurs, HARQ with soft combining is used to augment the performance of decoding.

We consider a system with $N$ UEs, indexed by $i$. Fig. 2 shows an example of the proposed periodic resource reservation for 4 users. As we consider that the environment is slowly changing inside the factory, the average SNRs are constant for a relatively long time. Therefore, the reservation is performed identically in each cycle. As the services are delay-constrained, we only allow one retransmission. Our model can be easily extended to a larger (but limited) number of retransmissions. There is clearly a tradeoff between the number of resources reserved for the first transmission and for retransmissions. Indeed, a more robust MCS ensures fewer retransmissions, and then less reservation of resources for HARQ, but has a lower spectral efficiency leading to more reserved resources for the first transmission. There is an optimal tradeoff to seek. In this section, we start by considering that the MCS is chosen for each UE and proposing the corresponding resource allocation scheme. The optimal tradeoff is studied in Section IV-C.

\footnotetext{
${ }^{1} 4 \mathrm{G}$ subchannels are of $180 \mathrm{kHz}$, each composed of 12 consecutive and equally spaced Orthogonal Frequency-Division Multiplexing (OFDM) subcarriers. Different subcarrier spacings are defined for $5 \mathrm{G}$, but our model is sufficiently generic to cover the different cases.
} 
Fig. 2. Periodic Resource Block reservation

\section{A. Resource Allocation for first transmissions}

The first step is to estimate the number of RBs for transmitting one applicative packet of URLLC service. This number is determined by the MCS and the structure of one RB.

First of all, we should calculate the spectral efficiency, $\eta$, measured in bit/s/Hz, of a specific MCS. $\eta$ refers to the information rate that can be transmitted over a given bandwidth and is given by:

$$
\eta=\eta_{c} \eta_{s} \log _{2} M
$$

where $M$ denotes modulation order, $\eta_{c}$ denotes code efficiency and $\eta_{s}$ denotes efficiency of signaling.

Suppose that the size of an applicative packet is $b$ bits. The bandwidth for a RB is represented by $\omega$. And the TTI is $\tau$. The number of physical RBs, $R$, for transmitting an applicative packet is:

$$
R=\left\lceil\frac{b}{\eta \tau \omega}\right\rceil
$$

In the following, we will define $\mu_{i}$ as the chosen MCS for $\mathrm{UE} i$. The required RBs for the first transmission of a packet of $\mathrm{UE} i$ is then:

$$
R_{i}=g\left(\mu_{i}\right)=\left\lceil\frac{b}{\eta\left(\mu_{i}\right) \tau \omega}\right\rceil
$$

We also define the users MCS selections vector $\vec{\mu}=$ $\left[\mu_{1}, \mu_{2}, \ldots, \mu_{N}\right]$.

\section{B. Resource Allocation for retransmissions}

Since the MCS stays the same in retransmission due to HARQ, the required number of RBs is exactly the same as the previous transmission for each UE. However, it is not optimal to reserve resources of retransmissions of all UEs as the loss probability is usually low, leading to a waste of resources for full reservation. We suppose that the amount of resources reserved for retransmissions is $H$. Therefore, we have as objectives are to determine the loss probability knowing the users MCS selections, $\vec{\mu}$, and the resources reserved for retransmissions $H$ and to derive optimal resource allocation, as presented in the next section.

\section{OPTIMAL JOINT MCS SELECTION AND RESOURCE ALLOCATION}

In order to obtain the optimal scheme, we start by computing the performance under a given users MCS selection and resource reservation. We then derive the optimal scheme under performance constraints.

\section{A. Distribution of the number of losses}

To calculate the probability mass function of lost RBs, every user's transmission process should be considered. The relationship between loss rate of $U E_{i}$ and MCSs could be described by an error rate function $f$. The number of needed RBs for retransmission can be modeled as a Bernoulli random variable $\epsilon_{i}$ :

$$
\begin{gathered}
\epsilon_{i}= \begin{cases}R_{i} & \delta_{i} \\
0 & 1-\delta_{i}\end{cases} \\
\text { with } \delta_{i}=f\left(\mu_{i}\right)
\end{gathered}
$$

where $\delta_{i}$ is the error probability of the first transmission for user $i$ and it is related with its MCS choice. This relationship is defined by function $f$.

We can now compute the distribution of the overall number of retransmissions. Let $X=\sum_{i=1}^{N} \epsilon_{i}$ be the number of needed RBs for retransmissions, we need to compute:

$$
P(x, \vec{\mu})=P(X=x \mid \vec{\mu})
$$

The lost number of RBs is a sum of independent weighted Bernoulli trials that are not identically distributed. In other words, it is the probability distribution of the total number of lost RBs in a sequence of $N$ independent experiments with error probabilities $\delta_{1}, \delta_{2}, \ldots, \delta_{N}$. The distribution function is necessary to decide how many RBs should be reserved for HARQ for a given target reliability. To simplify notations, let $\alpha_{i}=\epsilon_{i} / R_{i}$ to be the first transmission result for $U E_{i}\left(\alpha_{i}=1\right.$ means a failed transmission), and

$$
\vec{\alpha}=\left(\alpha_{1}, \alpha_{1}, \ldots, \alpha_{N}\right) .
$$

The state of all possible events is represented by:

$$
\mathcal{A}=\left\{\left(\alpha_{1}, \alpha_{1}, \ldots, \alpha_{N}\right) \mid \alpha_{i} \in\{1,0\}\right\}
$$

The probability of event $\vec{\alpha}$ could be expressed with error rate of each user:

$$
q(\vec{\alpha})=\prod_{i}^{N}\left[\delta_{i} \mathbb{1}_{\alpha_{i}=1}+\left(1-\delta_{i}\right) \mathbb{1}_{\alpha_{i}=0}\right]
$$

The indicator function $\mathbb{1}_{E}$ is equal to 1 if condition $E$ is verified and to 0 otherwise. The probability mass function of lost RBs is shown as below:

$$
\begin{gathered}
P(x, \vec{\mu})=\sum_{\vec{\alpha} \in \mathcal{A}} \mathbb{1}_{\{R(\vec{\alpha})=x\}} q(\vec{\alpha}) \\
\text { with } \quad R(\vec{\alpha})=\sum_{i=1}^{N} \alpha_{i} R_{i}
\end{gathered}
$$

Once we have the probability mass function of lost RBs, the cumulative distribution function could be easily derived (equation(6) ) and the number of RBs to be reserved will be calculated with final loss constraint.

$$
F_{X}(x)=\sum_{x_{i} \leq x} P\left(x_{i}, \vec{\mu}\right)
$$




\section{B. Reliability computation}

To evaluate the reliability of our resource allocation mechanism, we have to consider two possible events for loss as follows. First, if the number of needed resources for retransmissions is larger than $H$, some of the lost packets cannot be retransmitted, leading to a definite loss. Second, even if there is enough space for a retransmissions, the retransmission may fail again. Note also that, for the first event, i.e., when there is no enough space to accommodate all retransmissions, several policies are possible for selecting the packets to be dropped. We here adopt the policy that accommodates the highest number of retransmissions. For instance, if the UEs are ordered so that $R_{i} \leq R_{j}$ if $i<j$ and the vector of losses is $\vec{\alpha}$, the packets of UE 1 to $I^{*}(\alpha, H)$ are served with $I^{*}(\alpha, H)$ the largest index so that $\sum_{i=1}^{I^{*}} R_{i} \alpha_{i}<H$.

The following equation calculates the final error rate for UE $i$ :

$e_{i}(\vec{\mu}, H)=\sum_{\vec{\alpha} \in \mathcal{A}} \mathbb{1}_{\left\{\alpha_{i}=1\right\}} q(\vec{\alpha})\left\{\mathbb{1}_{i \leq I^{*}(\vec{\alpha}, H)} \delta_{i}^{(1)}+\mathbb{1}_{i>I^{*}(\vec{\alpha}, H)}\right\}$

where $\delta_{i}^{(1)}$ denotes the error rate during the retransmission for user $i$ after retransmission and decoding. The MCS-dependent error rates for first and second transmissions $\delta_{i}$ and $\delta_{i}^{(1)}$ are calculated using link level simulations as will be explained next.

\section{Optimal scheme}

Above we have developed a framework for evaluating the performance (in terms of packet losses) for URLLC services using periodic scheduling. The performance was derived for a set of selected MCSs and for a given reservation of resources for retransmissions. In this section we present an optimization framework that minimizes the overall reserved resources while achieving the target requirements. A two stage optimization problem is proposed. First, for given MCSs, the minimal reservation for HARQ is derived. Based on this result, a second optimization problem is formulated for the selection of MCSs.

1) Optimal resource allocation knowing the users MCS selection: For a predetermined selection of MCSs, the optimal reservation of resources for retransmissions (denoted by $H^{*}(\vec{\mu})$ ) is the smallest $H$ so that the following set of constraints is verified:

$$
e_{i}(\vec{\mu}, H) \leq \Theta, \quad \forall i \in[1, N]
$$

where $\Theta$ denotes the reliability target and $e_{i}(\vec{\mu}, H)$ the per user packet loss rate of as expresed in equation (7). Since $H$ is an integer and $e_{i}(\vec{\mu}, H) \geq e_{i}(\vec{\mu}, H+1)$, we can simply do a binary search with minimum values 0 and the maximum resource blocks and find the optimal $H^{*}(\vec{\mu})$. This search is very efficient and the complexity is logarithmic in the maximum number of RBs possible.
2) Global optimization: Once the minimal resource allocation for a given MCS selection is computed, we can derive the global optimal MCS for minimizing overall resource consumption:

$$
\vec{\mu}^{*}=\underset{\vec{\mu}}{\operatorname{argmin}} \sum_{i=1}^{N} R_{i}\left(\mu_{i}\right)+H^{*}(\vec{\mu})
$$

This can be done by exhaustively searching over all possible MCS combinations.

\section{NUMERICAL RESUlts}

We consider a system where the machine type equipments are randomly and uniformly distributed throughout the factory. The environment is assumed to be controlled, including interferences, so that the average SNRs are known (or estimated on a long term). We consider for illustration three classes of SNR $(5 \mathrm{~dB}, 10 \mathrm{~dB}$ and $15 \mathrm{~dB}$ ). We consider a traffic with small applicative packets generated periodically, with one packet generated every $20 \mathrm{~ms}$. The simulation parameters are listed in Table I.

\section{A. Link level simulations}

Optimal resource allocation analysis is performed using a link-level simulator where different modulations (64QAM, 16QAM, 4QAM), channel coding schemes (turbo, polar) and MIMO schemes are implemented, along with HARQ with Chase combining. Table II provides the results of the link level simulations, in terms of packet loss after the first transmission and after retransmission, for each MCS. As the reliability depends on the MCS combination $\vec{\mu}$ (equation (7)), we define in Table III the different possible combinations, eliminating combinations for which it is obvious that the target reliability cannot be achieved.

TABLE I

SIMULATION ASSUMPTIONS

\begin{tabular}{|c|c|}
\hline Applicative packet size & 64 bytes \\
\hline Number of UE & 15 \\
\hline Number of subcarrier & 12 \\
\hline Subcarrier spacing & $15 \mathrm{kHz}$ \\
\hline TTI & $0.5 \mathrm{~ms}$ \\
\hline Bandwidth of RB & $180 \mathrm{kHz}$ \\
\hline Efficiency of signaling & $11 / 14$ \\
\hline Reliability target & $10^{-5}$ \\
\hline
\end{tabular}

\section{B. Performance evaluation}

For each MCS combination, defined in Table III, we compute the amount of resources needed for the first transmission and for retransmissions under constraints (8). Figure 3 shows these allocations for the different combinations of Table III, and in the same time illustrates the global optimal solution of problem (9). 
TABLE II

PACKET ERROR RATE FOR DIFFERENT MCSS AND SNRS. THE FIRST VALUE CORRESPONDS TO THE ERROR RATE AFTER THE INITIAL TRANSMISSION AND THE SECOND CORRESPONDS TO THE HARQ ERROR RATE.

\begin{tabular}{|c|c|c|c|}
\hline & $5 \mathrm{~dB}$ & $10 \mathrm{~dB}$ & $15 \mathrm{~dB}$ \\
\hline $\begin{array}{c}\text { 1. Non coded } \\
\text { CP-OFDM 64QAM }\end{array}$ & $1: 1$ & $1: 1$ & $0.99: 0.99$ \\
\hline $\begin{array}{c}\text { 2. Alamouti2 } 2 \\
\text { OFDM 64QAM }\end{array}$ & $1: 1$ & $1: 1$ & $1: 0.96$ \\
\hline $\begin{array}{c}\text { 3. C(7,5) Alamouti2*2 } \\
\text { OFDM 64QAM }\end{array}$ & $1: 1$ & $0.99: 0.96$ & $0.4: 0.11$ \\
\hline $\begin{array}{c}\text { 4. Non coded } \\
\text { CP-OFDM 16QAM }\end{array}$ & $1: 1$ & $0.991: 0.96$ & $0.8: 0.47$ \\
\hline $\begin{array}{c}\text { 5. Alamouti2 } 2 \\
\text { OFDM 16QAM }\end{array}$ & $1: 1$ & $0.98: 0.84$ & $0.45: 0.06$ \\
\hline $\begin{array}{c}\text { 6. C(7,5) Alamouti2*2 } \\
\text { OFDM 16QAM }\end{array}$ & $1: 1$ & $0.91: 0.53$ & $0.17: 0.007$ \\
\hline $\begin{array}{c}\text { 7. Turbo Alamouti } \\
\text { OFDM 16QAM }\end{array}$ & $0.99: 0.86$ & $0.83: 0.7$ & $0.14: 0.099$ \\
\hline $\begin{array}{c}\text { 8. MIMO4*4 } \\
\text { OFDM 16QAM }\end{array}$ & $0.97: 0.85$ & $0.5: 0.2$ & $0.012: 0.002$ \\
\hline $\begin{array}{c}\text { 9. Polar Alamouti } \\
\text { OFDM 16QAM }\end{array}$ & $0.93: 0.54$ & $0.17: 0.009$ & $0.006: 1 \mathrm{e}-6$ \\
\hline $\begin{array}{c}\text { 10. No coded } \\
\text { CP-OFDM 4QAM }\end{array}$ & $0.97: 0.8$ & $0.62: 0.32$ & $0.26: 0.04$ \\
\hline $\begin{array}{c}\text { 11. Alamouti2*2 } \\
\text { OFDM 4QAM }\end{array}$ & $0.91: 0.41$ & $0.16: 0.007$ & $0.005: 5 \mathrm{e}-5$ \\
\hline $\begin{array}{c}\text { 12. C(7,5) Alamouti2*2 } \\
\text { OFDM 4QAM }\end{array}$ & $0.34: 0.67$ & $0.02: 0.0003$ & $0.0004: 4 \mathrm{e}-5$ \\
\hline $\begin{array}{c}\text { 13. Turbo Alamouti } \\
\text { OFDM 4QAM }\end{array}$ & $0.18: 0.14$ & $0.08: 0.05$ & $1 \mathrm{e}-5: 9 \mathrm{e}-6$ \\
\hline $\begin{array}{c}\text { 14. Polar Alamouti } \\
\text { OFDM 4QAM }\end{array}$ & $0.1: 0.003$ & $0.004: 1 \mathrm{e}-5$ & $1 \mathrm{e}-3: 1 \mathrm{e}-6$ \\
\hline $\begin{array}{c}\text { 15. MIMO4*4 } \\
\text { OFDM 4QAM }\end{array}$ & $0.017: 1 \mathrm{e}-5$ & $1 \mathrm{e}-5: 1 \mathrm{e}-7$ & $1 \mathrm{e}-5: 1 \mathrm{e}-7$ \\
\hline
\end{tabular}

TABLE III MCS COMBINATIONS

\begin{tabular}{|c|c|c|c|}
\hline MCS combination & MCS for 5dB & MCS for 10dB & MCS for 15dB \\
\hline 1 & 15 & 14 & 9 \\
\hline 2 & 15 & 14 & 11 \\
\hline 3 & 15 & 14 & 12 \\
\hline 4 & 15 & 14 & 13 \\
\hline 5 & 15 & 14 & 14 \\
\hline 6 & 15 & 14 & 15 \\
\hline 7 & 15 & 15 & 9 \\
\hline 8 & 15 & 15 & 11 \\
\hline 9 & 15 & 15 & 12 \\
\hline 10 & 15 & 15 & 14 \\
\hline 11 & 15 & 15 & 15 \\
\hline 12 & 15 & 15 & \\
\hline
\end{tabular}

Following Figure 3, the optimal combination of MCS is No.7, which corresponds to using MIMO 4×4 OFDM 4QAM for UEs with SNR $5 \mathrm{~dB}$ or $10 \mathrm{~dB}$ and Polar Alamouti OFDM 16QAM for UEs with $15 \mathrm{~dB}$ (see Table III).

The trade-off between robustness of MCS and error probability can also be observed from Figure 3. For example, the last MCS combination No.12 is the most robust one with MIMO $4 \times 4$ OFDM 4QAM for all UEs. It requires $120 \mathrm{RBs}$ for first transmission, which is more than what is required by combination No.7, and 16 RBs for HARQ, which is less

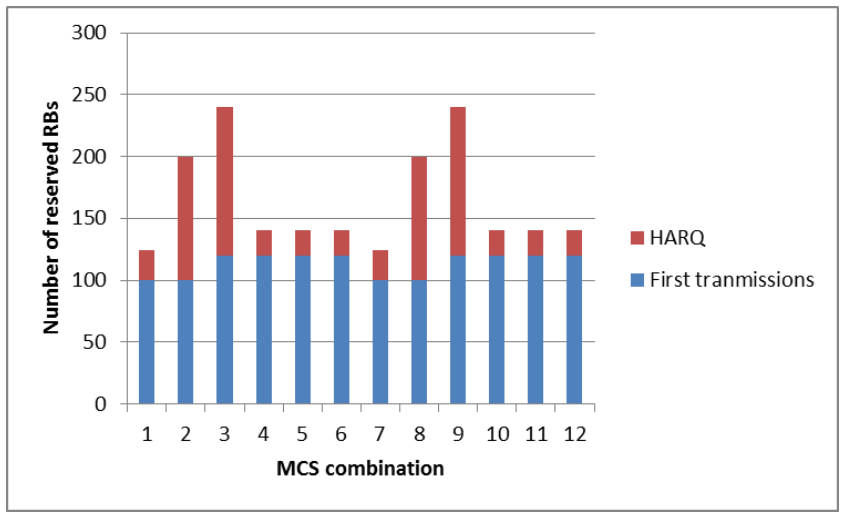

Fig. 3. Number of RBs to be reserved for different MCS combination with $\Theta=10^{-5}$

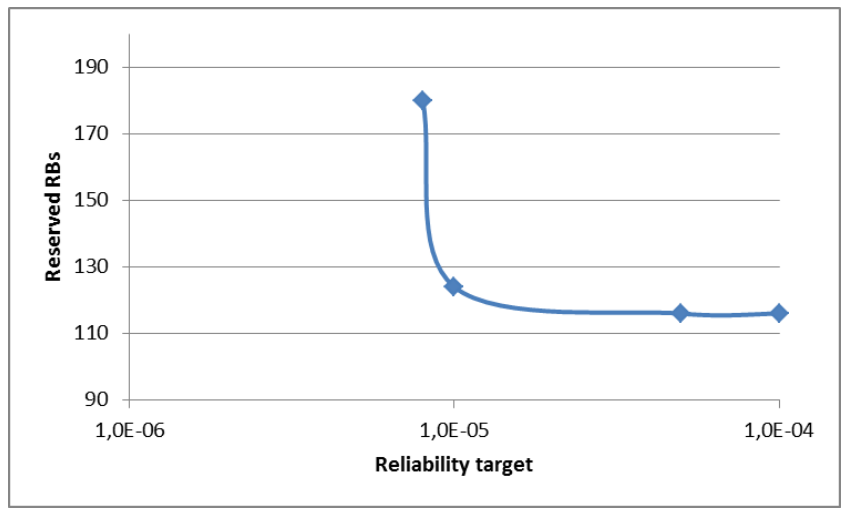

Fig. 4. Number of RBs to be reserved for different reliability targets for a mix of users with good and bad radio conditions.

than No.7. We could conclude that high modulation order is useful for users in good radio conditions, where it may be used to improve spectral efficiency. As for users with bad radio conditions, robustness is more important.

\section{Impact of reliability requirement}

We now turn to the evaluation of the impact of the target reliability on the resource allocation. In Figure 4 it can be observed that a tighter constraint on reliability increases the required resources. However, the system is constrained with users having the lowest SNR, and an error rate lower than $10^{-5}$ is not achievable. Figure 5 shows the same result but for a system where all users have good channel conditions $(15 \mathrm{~dB})$, showing how targets as stringent as $10^{-7}$ can be reached, provided that the system is over-dimensioned (e.g., by densification).

\section{Impact of latency requirement}

We now turn to the evaluation of the impact of the proposed scheme on the latency. For doing so, we need to understand the different components of the latency in a practical 5G deployment. We consider the system parameters of [9] for $5 \mathrm{G}$ new radio. In particular, we consider a short TTI of $0.5 \mathrm{~ms}$ and a Frequency Division Duplexing (FDD) system for minimizing 


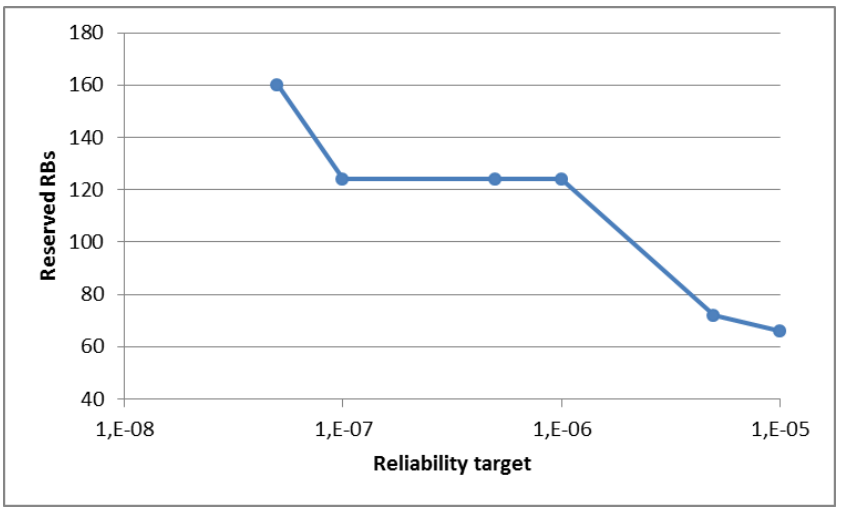

Fig. 5. Number of RBs to be reserved for different reliability targets when all UEs have a good SNR $(15 \mathrm{~dB})$.

latency. According to [9], in such a system, the minimum delay before a HARQ transmission can be scheduled is equal to $d=1.5 \mathrm{~ms}$, due to the packet processing time and the NACK transmission/reception delay. For an MCS combination $\vec{\mu}$ and the corresponding optimal HARQ resource allocation $H^{*}(\vec{\mu})$, the maximal delay for a packet that is retransmitted is given by:

$$
D(\vec{\mu})=\left\lceil\frac{H^{*}(\vec{\mu})+\sum_{i} R_{i}\left(\mu_{i}\right)}{r}\right\rceil+d+\left\lceil\frac{H^{*}(\vec{\mu})}{r}\right\rceil
$$

where $r$ be the number of available RBs per TTI (that depends on the amount of available spectrum for the URLLC applications).

Figure 6 shows the required amount of spectrum for different application delay constraints, obtained by computing the minimal value of $r$ whose corresponding delay in equation 10 is lower than the delay target (knowing that one RB corresponds to $180 \mathrm{KHz}$ ). The figure shows that, when the application is very delay-sensitive, the amount of resources to be reserved is very large, but this amount reduces rapidly when the delay requirements are relaxed. For illustration, for a very stringent delay requirement of $2.5 \mathrm{~ms}$, the resource consumption is $36 \mathrm{MHz}, 22,3 \mathrm{MHz}$ and $24,5 \mathrm{MHz}$ for MCS combinations No.2, No.7 (optimal) and No.12, respectively. This gap reduces when the latency target is lower, but the optimal MCS combination is still the same as computed for the target reliability constraint).

\section{CONCLUSION}

In this article, we have developed a joint MCS selection and resource reservation scheme for reaching stringent latency and reliability targets in factory-like environments, while ensuring minimal resource consumption. We considered a system with quasi-static users and reserve resources for first transmissions and a limited set of retransmissions and formulated a twostage optimization problem for selecting the optimal MCS combination. Our simulation results illustrate the optimal scheme and the impact of different reliability and latency targets on the resource reservation. As a future work, we aim at extending our model to multiple retransmissions (within

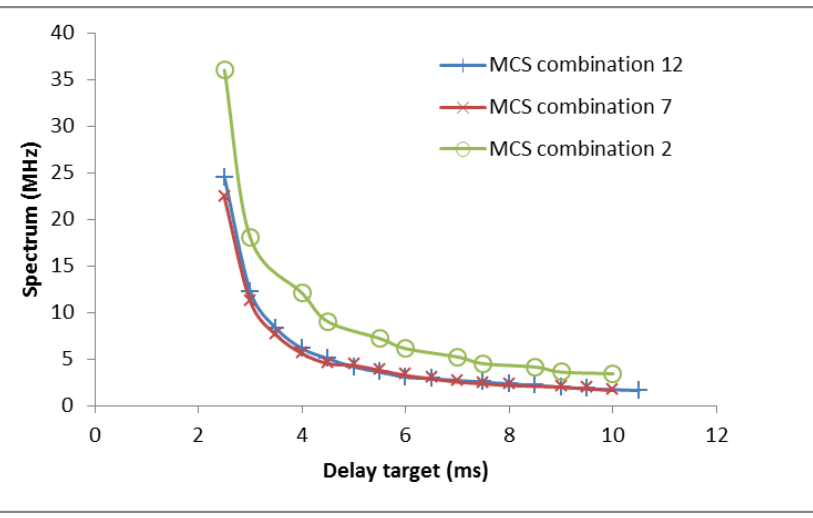

Fig. 6. Impact of delay constraint on the amount of spectrum to be reserved.

the latency budget) and to more distributed schemes where collisions between retransmissions may occur.

\section{REFERENCES}

[1] NGNM Alliance, "5G white paper", 2015

[2] 5G PPP white paper, "5G Empowering Vertical Industries", Mobile World Congress 2016.

[3] H. Zhang, P. Soldati, and M. Johansson, Time-and channel-efficient link scheduling for convergecast in WirelessHART networks, in Proceedings of the 13th IEEE International Conference on Communication Technology (ICCT), 2011.

[4] N. A. Johansson, Y.-P. E. Wang, E. Eriksson, M. Hessler, "Radio Access for Ultra-Reliable and Low-Latency 5G Communications," IEEE International Conference on Communications (ICC) Workshop on 5G \& Beyond - Enabling Technologies and Applications, June 2015.

[5] O. N. C. Yilmaz, Y.-P. E. Wang, N. A. Johansson, N. Brahmi, S. A. Ashraf, J. Sachs "Analysis of Ultra-Low Latency and Ultra-Reliable 5G Communication for a Factory Automation Use Case," IEEE International Conference on Communications (ICC) Workshop on 5G \& Beyond Enabling Technologies and Applications, June 2015.

[6] N. Brahmi, O. N. C. Yilmaz, K. W. Helmersson, S. A. Ashraf and J. Torsner, "Deployment Strategies for Ultra-Reliable and Low-Latency Communication in Factory Automation," 2015 IEEE Globecom Workshops (GC Wkshps), San Diego, CA, 2015, pp. 1-6.

[7] S. S. Craciunas, R. S. Oliver and V. Ecker, "Optimal static scheduling of real-time tasks on distributed time-triggered networked systems," Proceedings of the 2014 IEEE Emerging Technology and Factory Automation (ETFA), Barcelona, 2014, pp. 1-8.

[8] S. S. Craciunas, R. S. Oliver, M. Chmelik and V. Ecker, "Scheduling Real-Time Communications in IEEE802.1Qbv Time Sensitive Networking," Proceedings of the 24th International Conference on Real-Time Networks and Systems, Brest, FR, 2016, pp. 183-192.

[9] 3GPP Tdoc R2-1711550, 'User plane latency in NR', 2017.

[10] 3GPP TS 22.261-g20 Service requirements for the 5G system, Rel 16, Dec 2017. 